\title{
No association between SCN9A gene rs6746030 polymorphic variant and psychoactive drug addiction
}

\author{
Poltavskaya E.*, Savochkina D. \\ Mental Health Research Institute of Tomsk NRMC, Tomsk, Russia \\ *e-mail:egboyarko@mail.ru
}

Key words: SCN9A, gene polymorphism, addiction, psychoactive substance

Motivation and Aim: SCN9A gene encodes voltage-gated sodium channel NaV1.7, which expressed in the nervous system. Shown that NaV1.7 is present within neurons in the hypothalamic supraoptic nucleus, specifically within vasopressin- and oxytocinproducing magnocellular neurosecretory neurons [1]. Alterations to the development of the oxytocin system plays a pivotal role in the regulation of social behaviours and emotion [2]. Dysregulation of this system also contributes to increased susceptibility to develop drug addiction. The aim of this study was to study the possible association of rs6746030 polymorphism with psychoactive drug addiction in Russian population of Siberian region.

Methods and Algorithms: The groups for study were formed on the basis of psychoneurologic dispensaries (patients) and higher schools (healthy persons) in two cities of West Siberia (Russia). We examined 213 persons - 106 participants with psychoactive drug (PAD) addiction (F1x.2 according to ICD-10), and 107 healthy person (control group). Genotyping of rs6746030 SCN9A has been carried out using ABI StepOne Plus with TaqMan1 Validated SNP Genotyping Assay (Applied Biosystems). The Hardy-Weinberg equilibrium of genotypic frequencies was tested by the chi-square test. Statistical analyses were performed using SPSS software, release 21, for Windows; $\mathrm{P}<0.05$ was considered as significant.

Results: The genotype distribution of rs6746030 SCN9A polymorphism were in agreement with Hardy-Weinberg equilibrium in patient group and in control group. Comparison of genotypes frequencies of SCN9A gene rs6746030 polymorphism in groups of patients with PAD addiction and healthy persons has not revealed statistically significant difference. The genotypes distribution has been shown as follows: GG (69.8 and $73.8 \%$ ), AG (27.4 and $22.4 \%$ ), AA (2.8 and 3.7\%) in patients with PAD addiction and healthy persons, respectively.

Conclusion: Thus, there is no association between SCN9A gene rs6746030 polymorphic variant and PAD addiction in Russian population of Siberian region.

Acknowledgements: This work has been completed under support of RFBR research project No. 17-36-01113.

\section{References}

1. Black J.A. et al. NaV1.7: stress-induced changes in immunoreactivity within magnocellular neurosecretory neurons of the supraoptic nucleus. Mol. Pain. 2013;8(9):39.

2. Baracz S.J. et al. The impact of early life stress on the central oxytocin system and susceptibility for drug addiction: applicability of oxytocin as a pharmacotherapy. Neurosci Biobehav Rev. 2018; 30:pii:S0149-7634(18)30276-8. 